\title{
Non-muscle Invasive Bladder Cancer: Identifying Patients to Consider Timely, Initial Cystectomy
}

\author{
(1) George E. Koch, (1) Sam S. Chang \\ Vanderbilt University Medical Center, Clinic of Urology, Nashville, USA
}

\begin{abstract}
Non-muscle invasive bladder cancer is a heterogeneous disease with widely varying risks of recurrence and progression to muscle invasion. Risk stratification within current treatment guidelines are an attempt to address this heterogeneity. This heterogeneity stems from a number of pathologic characteristics as well as recurrence status. Radical cystectomy, while the definitive, curative therapy for muscle invasive disease, is not often utilized for non-muscle invasive disease, even when it may be a superior option to bladder-sparing therapy. A majority of American Urologists have been reported to defer cystectomy for less invasive therapies when it is a guideline driven option. Both cystectomy and bladder-sparing therapy have excellent oncologic outcomes, but very different morbidity and mortality profiles. Timely, initial cystectomy provides improved oncologic outcomes for patients with high-risk non-muscle invasive bladder cancer, as opposed to delayed cystectomy following prolonged, failed bladder-sparing therapy. Patient selection is paramount when considering timely, initial cystectomy, given the increased rates of morbidity and mortality. The heterogeneity of non-muscle invasive pathologic characteristics includes a number of factors that may help differentiate patients who would benefit from a timely, initial cystectomy as opposed to bladder-sparing therapy. A rapidly evolving understanding of bladder cancer biology may help improve risk stratification and further aid in the selection of patients for timely, initial cystectomy.
\end{abstract}

Keywords: Early cystectomy, high-risk bladder cancer, non-muscle invasive bladder cancer, timely cystectomy

\section{Introduction}

Bladder cancer burdens the healthcare system as the sixth most commonly diagnosed cancer in the United States (US), with the highest per patient cost of any cancer in the US $(1,2)$. These statistics are even more troublesome as the incidence and death rate of bladder cancer have remained remarkably unchanged from 1985 (20.7 and 4.7 per 100,000) to 2016 (19.4 and 4.4 per 100,000) based on the SEER Medicare Dataset (2).

Approximately $75 \%$ of bladder cancers are non-muscle invasive bladder cancer (NMIBC) at diagnosis (3). This predominance for non-muscle invasive disease complicates the treatment algorithm due to its heterogeneity of risk and treatment options. Treatment of NMIBC by the American Urological Association (AUA) Guidelines relies on risk stratification based on tumor characteristics and recurrence status. The applicability of these treatment algorithms has improved with the current guidelines by the introduction of, and emphasis on, risk re-stratification at every subsequent recurrence, treatment and transurethral resection of a bladder tumor (TURBT) (4).

\section{Treatment of NMIBC and Early Cystectomy}

Both recurrence and progression rates vary widely for low, intermediate and high-risk NMIBC, with the overall risk of recurrence as high as $70 \%$ within the first year, and the lifetime risk of progression to muscle invasive disease between 10-50\% $(5,6)$. This variability reflects the range of factors that contribute to the AUA risk stratification, namely the depth of tumor invasion, presence of lymphovascular invasion (LVI), concomitant carcinoma in situ (CIS), tumor size and location, tumor biology including a number of potential variants and recurrence status $(5,7,8,9,10)$. However, these factors represent an incomplete list, as molecular and genetic factors will help determine more effective therapeutic strategies on an individual basis $(11,12)$.

This heterogeneity within the NMIBC cohort, though complicating, emphasizes the need to categorize patients who may bear increased risk from bladder-sparing, intravesical therapy and thus may benefit from timely, initial, up front cystectomy. It should be noted that repeat TURBT, when indicated, is essential for appropriate risk stratification, and has been associated with decreased mortality and increased rates of tumor upstaging (13). Guideline-directed treatment of NMIBC includes complete resection of all visible tumor and risk-guided surveillance, and may include intravesical instillation of immunotherapy [Bacillus Calmette-Guerin (BCG)] or chemotherapy, re-resection of the primary tumor site, or radical cystectomy $(4,14)$. There is no argument that bladder-sparing techniques serve as the backbone 
of NMIBC therapy. Both induction and maintenance courses of intravesical BCG have been shown to decrease recurrence and progression of NMIBC when compared to TURBT alone or to TURBT plus intravesical chemotherapy $(15,16,17,18)$.

Regarding BCG for patients with high-grade invasion into the lamina propria NMIBC (T1), multiple retrospective analyses have demonstrated BCG's efficacy. In a series of 126 patients with T1 disease, Brake et al. (19) reported a recurrence-free rate of $86 \%$ at 53 months following 1 or 2 cycles of induction BCG. These successes were re-demonstrated by Novotny in a group of 63 patients who underwent a combination of induction and maintenance BCG regimens showing a recurrence in $25 \%$ and progression in $13 \%$ of patients. Canter also showed a decrease in progression to $8.6 \%$ with the addition of adjuvant BCG (20).

Given these results for BCG, and realizing the morbidity of radical cystectomy, up front bladder removal, even for high-risk NMIBC patient, is not often considered (21). This holds true for BCG refractory NMIBC, which carries an approximately $80 \%$ chance of treatment failure or progression, as evidenced by one survey in which $80 \%$ of American Urologists would not recommend a radical cystectomy for a patient with NMIBC refractory to two induction courses of BCG (22). This lack of adoption is likely multifactorial, but worrisome given the excellent, albeit retrospective data, supporting upfront, timely radical cystectomy versus delayed cystectomy following failed bladder-sparing therapy. Herr and Sogani (23) reviewed 90 patients with highrisk NMIBC who underwent cystectomy. Of the patients whose cystectomy was within 2 years of BCG initiation, 15-year cancerspecific survival was $69 \%$, versus $29 \%$ in those who underwent cystectomy more than 2 years after BCG initiation. While none of these patients underwent cystectomy prior to progression to muscle invasion, Jäger et al. (24) showed a similar result in strictly high-risk NMIBC patients, with a ten-year overall survival of $79 \%$ in patients who underwent cystectomy within 6-12 months of their initial TURBT as opposed to $62 \%$ for cystectomy after 12 months. Raj et al. (25) then compared Herr's original group to a more contemporary cohort that underwent early cystectomy for recurrent T1 disease, showing an improved disease-specific survival favoring early cystectomy, which was then echoed by Denzinger et al. (26) in a similar population. Denzinger et al. (26), Hautmann et al. (27) and Stöckle et al. (28) have all reported similar findings in patients who either underwent early radical cystectomy for high-risk non-muscle invasive disease versus delayed cystectomy following failure of bladder-sparing therapy, showing five-year disease-specific survivals of $90 \%$ compared to $62 \%$ and ten-year disease-specific survivals of 78$79 \%$ compared to $51 \%-65 \%$, favoring early cystectomy.

Given the excellent outcomes from both bladder-sparing BCG, and from timely, initial cystectomy, it is unsurprising that a recent meta-analysis showed mixed results regarding the differences in cancer-specific survival for bladder-sparing therapy versus timely cystectomy for patients with high-risk T1 NMIBC (29). In this analysis, cancer-specific survival showed no significant difference between upfront cystectomy versus intravesical therapy at 2, 5 and 10-years; it favored cystectomy at 15-years, however with significant variability of the data. Overall survival on the other hand favored bladder-sparing intravesical therapy at 2, 5 and 10 -years, with insufficient data for 15 years. It should be noted that these results reflect both a heterogeneous group of studies, and include complete responders to intravesical therapy, thus decreasing the power of the meta-analysis to detect differences between immediate versus delayed cystectomy. The true denominator of those who never received cystectomy and instead received alternative, likely ineffective, intravesical therapies is unknown and likely underestimated.

Outside of oncologic outcomes, any discussion of radical cystectomy should also take into account both the advantages of cystectomy's improved staging and the disadvantages of its increased morbidity and mortality. Accurate staging is paramount when discussing the potential treatment options available for bladder cancer given the strong evidence for radical cystectomy for muscle invasion (30). Unfortunately, staging is often not as technically straightforward as the dichotomy of "muscle invasive or not". More than $20 \%$ of patients initially diagnosed with T1 NMIBC have been found to be incorrectly staged on repeat TURBT (31). This is echoed by the rates of pathologic upstaging at the time of radical cystectomy, which have found previously T1 NMIBC patients to be reclassified as muscle-invasive in $25-50 \%$ of cases, with $15 \%$ of patients found to harboring lymph-node positive disease at the time of cystectomy for NMIBC $(32,33)$.

Radical cystectomy is not without risk, which may account for the reported reluctance to recommend extirpative therapy, even when indicated (22). Contemporary series have reported the 90-day morbidly of radical cystectomy to be between 27-60\% when genitourinary, gastrointestinal, infectious and wound related complications are considered, regardless of an open versus robotic surgical approach, with mortality often cited between $3-5 \%(21,34,35)$. Outside of the perioperative risk, there has been controversy regarding quality of life outcomes after radical cystectomy. Yang et al. (36) found both genitourinary and sexual outcomes to be negatively impacted by radical cystectomy over bladder-sparing therapy. Interestingly, these results were significant regardless of continent versus incontinent urinary diversion. On the other hand, Kulkarni et al. (37) examined quality-adjusted life expectancy and found upfront radical cystectomy to have better outcomes than radical cystectomy following failure of bladder-sparing therapy.

Considering both the advantages and disadvantages of radical cystectomy weighed against the effectiveness of bladdersparing therapy, discussing the criteria for choosing the NMIBC patient appropriate for a timely, upfront cystectomy is essential. This patient may be chosen before or following re-staging TURBT. Unsurprisingly, this question has long been the focus of multiple research groups in an attempt to optimize the NMIBC treatment pathway. Risk stratification and predictive modeling have both been employed to this end. AUA Guidelines serve as a framework for this discussion, categorizing patients based on the pathologic features of TURBT specimens and conferring "high risk" status on any specimen with high-grade lamina propria invasion, variant pathology, CIS, multifocality or large size of a high-grade tumor, high-grade recurrences/BCG failure or prostatic specimens and LVI (4). Both the European Organization for Research and Treatment of Cancer (EORTC) and the Club Urologico Español de Tratamiento Oncologico have devised predictive models to help improve risk stratification and thus 
guide treatment decisions for NMIBC. Unfortunately, neither of these have shown much practical use for the modern patient, as both cohorts included patients without routine re-staging TURBT, did not categorize based on LVI or variant histology, and overall did not follow current treatment algorithms $(6,38)$. These shortcomings were demonstrated in a multi-institutional external validation study showing both prediction tools to have poor discrimination between progression and recurrence, with low positive predictive values and a tendency to overestimate the risk of disease progression for high-risk NMIBC (39).

Lacking a contemporary tool to aid in the decision for timely, upfront cystectomy, tumor characteristics must be taken in turn. Substaging the level of invasion of the lamina propria, as defined by the current American Joint Committee on Cancer Staging (AJCCS), has been a focus of much research. Both identification of muscularis mucosa involvement as well as the depth of lamina propria invasion have been identified to carry prognostic value (40). When T1 disease is subclassified into "superficial" versus "deep" invasion, Holmang et al. (8) showed a significant difference in both progression ( $36 \%$ vs $58 \%$ ) and disease-specific mortality ( $23 \%$ vs $45 \%)$. Rouprêt et al. (41) reported a similar finding, showing improved recurrence-free, progression-free and cancer-specific survival for T1a over T1b NMIBC. Further subdividing $\mathrm{T} 1$ tumors into $\mathrm{T} 1 \mathrm{a}, \mathrm{T} 1 \mathrm{~b}$, or $\mathrm{T} 1 \mathrm{c}$ depending on the depth of invasion above, into, or beyond the muscularis mucosa, respectively, has also been consistently associated with increased progression and decreased survival for deeper T1 subclasses $(5,42)$. Extent of muscularis mucosa invasion has also been proposed as a subclassification of $\mathrm{T} 1$ disease, although the exact definition of "extent" has not yet been standardized. Indeed, the most recent update to the AJCCS guidelines recommends an attempt at quantification of lamina propria invasion, although no specific strategy or metric is defined (43). Even though muscularis mucosa involvement has previously been discussed as a potentially useful measurement to subcategorize T1 NMIBC, its adoption has not been widely implemented, as its identification is extremely inconsistent. Muscularis mucosa is often scattered and discontinuous, and its reported detection rate ranges from 6 to $76 \%(44,45)$. Using depth of invasion with a tangible, easily defined cutoff for microinvasion has been more readily adopted and shown improved prediction of progression, over the EORTC calculator, and an association with progressionfree survival, depending on the cut-off used $(10,40)$. Even more recently, aggregate linear length of invasive carcinoma has been reported as an independent predictor of progression to muscle invasion in a recent study by Leivo et al. (46). While it is clear that sub-classification of lamina propria invasion has prognostic value, and will likely someday factor more directly into the decision to recommend timely cystectomy over bladder-sparing therapy, the lack of widespread validation and adoption of a single, definitive strategy for sub-classifying pure T1 NMIBC limits its current clinical application.

LVI provides another pathologic characteristic with the potential to help differentiate the patient who may benefit from a timely, initial radical cystectomy for non-muscle invasive disease. For MIBC, the presence of LVI is well established as a poor prognostic factor, and has been associated with advanced stage, grade and lymph-node involvement as well as decreased recurrence- free survival, cancer-specific survival and overall survival $(47,48,49)$. For NMIBC, large, multicenter cohorts have also shown independent associations with recurrence-free survival, progression-free survival and cancer-specific survival, and have been further confirmed by a 4.000 patient meta-analysis $(50,51,52)$. Unfortunately, LVI proves difficult to implement as a widespread metric due to technical challenges. Certain tissue processing artifacts like tissue retraction can complicate the identification of LVI (53). Furthermore, LVI in TURBT specimens only represents a small sample of the total tumor burden and thus it may be missed, or conversely overestimated in the overall tumor burden. Accordingly, concordance between TURBT and cystectomy specimens ranges from $18-79 \%$ with a negative predictive value of $50-70 \%(54,55,56)$. However, even considering these technical barriers to standardization, a recent meta-analysis and systematic review found that LVI in NMIBC tripled the risk of progression to MIBC and doubled the risk of disease recurrence. Furthermore, this evidence was so strong that the authors recommended LVI for inclusion in the TNM staging criteria for bladder cancer (57).

At diagnosis, approximately $10 \%$ of bladder cancer patients are found to have CIS, which represents a high-risk form of NMIBC, regardless of whether it presents as a discrete lesion, or synchronous with other non-muscle invasive lesions (4). At the time of cystectomy, specimens with $\mathrm{ClS}$ are understaged in up to $48 \%$ of non-muscle invasive cases, and the risk of pathologic upstaging in T1 NMIBC with synchronous CIS is $55 \%$ compared to $6 \%$ in high-risk $\mathrm{T} 1$ disease without $\mathrm{CIS}(58,59)$. The rate of progression in the presence of CIS also poses a major risk for NMIBC patients. In one study of NMIBC patients undergoing BCG therapy, $81 \%$ of whom had CIS, $53 \%$ of patients showed disease progression while on BCG therapy (60). This has been reproduced multiple times, demonstrating a strong association between CIS and progression to muscle invasion in 40-80\% of cases $(61,62)$. CIS has also been associated with nodal metastases in $4-6 \%$ of patients, along with risk of development of nodal metastases in up to $12 \%$ of patients when followed out to 3 years after cystectomy $(63,64)$. Considering the high risk of both understaging and progression, the outcomes for radical cystectomy in patients with CIS-only disease are promising, with 3-year disease-free survival reported as high as $88 \%$ (63). Furthermore, Masood et al. (58) found 5-year cancer-specific survival to be $82 \%$ for CIS with concomitant T1 NMIBC. However, these results have been challenged in the era of neoadjuvant chemotherapy with Amini et al. (65) reporting no difference in oncologic outcomes for patients with CIS versus those without CIS, when adjusted for stage.

Variant histologies of NMIBC represent pathologic subsets that fall into the high-risk category in the AUA risk stratification table, accounting for $7-10 \%$ of new bladder cancer diagnoses $(4,66)$. These include micropapillary, nested, plasmacytoid, neuroendocrine and sarcomatoid variants, each of which is thought to have its own tumor biology (67). One of the major concerns regarding variant histology is the relative rarity with which they are encountered as compared to pure urothelial carcinoma, and thus the lack of consistency regarding their identification and staging. Variant histologies go unrecognized in up to $44 \%$ of cases in one study of community pathologists 
(68). This may also play a factor in the $30-60 \%$ rate of understaging in variant histology, which represents up to triple the rate of pure urothelial carcinoma understaging $(69,70)$. Irrespective of difficulties with identification, variant histologies are considered more aggressive than pure urothelial carcinoma. Variant histologies present as muscle-invasive disease in up to three quarters of cases, as opposed to urothelial carcinoma that presents as non-muscle invasive in $70 \%$ of cases (71). Furthermore, variant histologies are associated with higher rates of locally advanced disease (72). The presence of a histologic variant has also been shown to be an independent predictor of nodal metastatic disease and decreased survival (73). Nested variant urothelial carcinoma has a propensity to progress to muscle-invasive disease more quickly than pure urothelial carcinoma, with a $54 \%$ rate of upstaging to muscle-invasive or metastatic disease after early cystectomy, even after thorough restaging TURBT showing CT1 disease (74). Regardless of the increased aggressiveness of variant histologies, it is important to note that intravesical therapy shows reduced effectiveness when compared to pure urothelial carcinoma, although this should be considered on a variant by variant basis. Kamat et al. (69) reported that up to $89 \%$ of patients with variant histology do not respond to intravesical therapy, with $67 \%$ actually progressing on BCG. This same cohort was then updated, showing that in patients with micropapillary variant, 5-year disease specific survival was $100 \%$ following upfront radical cystectomy versus $60 \%$ with BCG (75). A larger review of almost 900 patients with CT1 micropapillary bladder cancer showed no significant survival benefit for early cystectomy over bladder preserving therapy (76). It should be noted as well that the benefit of neoadjuvant chemotherapy is not quite clear, with a Vetterlein et al. (77) demonstrating improved rates of non-organ confined disease for many common histologic variants, but a survival benefit for only neuroendocrine variant.

As mentioned previously, in this age of personalized medicine, molecular and genomic classifications of bladder cancer are becoming critical in the considerations of potential patient management, although they have not yet been included in current risk stratifications. Biomarkers including p53, pRB and Ki-67 have all been associated with both increased recurrence and decreased survival for MIBC after radical cystectomy $(78,79)$. For NMIBC, while early trials had conflicting results, newer evidence is emerging that suggests that recurrence may be predicted, and prognostic models improved, by molecular marker data $(53,80,81,82,83,84)$.

Epigenetics, including oncogene activation, chromosomal alterations, tumor suppressor loss and cell-cycle regulation, has been associated with non-invasive tumor progression, which may serve as an indicator of patients who would benefit from timely, initial cystectomy $(85,86,87)$. Mutation of the tumor suppresser gene p53 has been shown in many studies to be associated with tumor progression, although there is some conflicting evidence $(88,89)$. Fibroblast growth factor receptor 3 (FGFR3) mutations, found to be present in up to $88 \%$ of lowgrade papillary lesions but only $16 \%$ of high-grade T1 tumors, have been associated with a lower rate of progression, recurrence and disease-specific survival (90). RNA sequencing has also be implicated as a possible classifier of NMIBC, showing the ability to predict progression and recurrence (91). Furthermore, combining panels of molecular markers has further improved risk stratification for NMIBC. Combinations of FGFR3 mutation status with $\mathrm{Ki}-67$ expression have been associated with progression, as well as shown significant improvement in the accuracy of the EORTC risk calculator $(92,93)$. Tissue microarrays using a large number of genetic markers including p53, pRB, p21 and p27, as well as one using cyclin D1, MCM7, TRIM29 and UBE2C, have not only shown an association with tumor progression, but have also provided a continuum on which the number of marker alterations correlates with the probability of progression $(86,94)$. While these various biomarkers have been shown to improve prognostication, they are not yet widely available in clinical practice.

The highest yield application of the increasing genetic and epigenetic knowledge base of bladder cancer may be the identification of two distinct molecular subtypes of NMIBC, namely the basal and luminal subtypes $(11,12,95)$. The luminal subtype tends to have more papillary features, rich in FGFR3, whereas the basal subtype are often associated with squamous and sarcomatoid features and are often found to be metastatic at diagnosis $(11,95)$. These new classifications may provide not only a more robust risk stratification model, but also a framework for increasingly targeted therapeutic algorithms (96).

Furthermore, bladder-sparing therapy is also likely to expand beyond the current intravesical options based on the increasingly deepening understanding of the basal and luminal subtypes $(97,98)$. Basal type tumors, for example, respond well to immunotherapy given their concentration of programmed death ligand-1 T-cells as well as EGFR inhibitors $(12,99,100)$. The potential for both improved risk stratification, as well as individualized patient care, could signal a paradigm shift in the approach to both MIBC and NMIBC. This is most clearly evidenced by the 25 ongoing clinical trials in the US for NMIBC (101).

\section{Conclusion}

The evidence for the oncologic benefit of cystectomy in the NMIBC setting is quite clear. However, the morbidity of cystectomy, both in the short-term perioperative period and in the long-term follow-up period, must be considered. Unfortunately, the data suggests that radical cystectomy for NMIBC is not as effective following ineffective, prolonged intravesical therapy. When choosing appropriate candidates, decisions about their treatment plans should follow repeat transurethral resections, when indicated, and their surgical fitness should be considered. Patients with non-invasive tumors not amenable to transurethral resection, regardless of risk stratification, should be considered for initial cystectomy. Those with tumors amenable to complete visual transurethral resection in patients fit for surgery, with tumor pathology reflecting pure urothelial carcinoma with high-risk characteristics like lamina propria invasion on repeat resection, deeper or more extensive lamina propria invasion, LVI, CIS or patients with histologic variants should all strongly be considered for a timely, initial cystectomy. As patients accumulate more of these risk factors, they should be considered increasingly stronger candidates 
for timely, initial cystectomy. Genetic, epigenetic and genomic risk factors are paramount to the future of bladder cancer risk stratification and treatment, for both NMIBC and MIBC, and will likely add more variables to consider in the decision for bladdersparing therapy versus timely, initial cystectomy for NMIBC.

\section{Acknowledgements}

Publication: The results of the study were not published in full or in part in form of abstracts.

Contribution: There is not any contributors who may not be listed as authors.

Conflict of Interest: No conflict of interest was declared by the authors.

Financial Disclosure: The authors declared that this study received no financial support.

\section{Ethics}

Peer-review: Externally and internally peer-reviewed.

\section{Authorship Contributions}

Concept: S.S.C., Design: G.E.K., Data Collection and Processing: G.E.K., Analysis and Interpretation: G.E.K., Literature Search: G.E.K., Writing: G.E.K.

\section{Short Quiz}

\section{1- Which of the statements below is not correct.}

A) Contemporary series have reported the 90-day morbidly of radical cystectomy to be between $27-60 \%$

B) More than $20 \%$ of patients initially diagnosed with T1 NMIBC have been found to be incorrectly staged on repeat TURBT.

C) Both genitourinary and sexual outcomes are equally impacted by radical cystectomy and bladder-sparing therapy.

D) Muscularis mucosa is often scattered and discontinuous, and its reported detection rate ranges from 6 to $76 \%$.

E) For NMIBC, large, multicenter cohorts have also shown independent associations with recurrence-free survival, progression-free survival and cancer-specific survival

Answer: C

2- Which of the genetic factors below has not a study showing its effect on NMIBC
A) p53
B) $p R B$
C) $\mathrm{p} 21$
D) p27
E) p99

Answer: $\mathrm{E}$
3- Which of the factors below does not contribute to the AUA risk stratification
A) Lymphovascular invasion (LVI),
B) Concomitant carcinoma in situ (CIS),
C) Tumor size
D) Cystoscopic appearance of the tumor
E) Tumor location

Answer: D

\section{References}

1. Botteman $M F$, Pashos $C L$, Redaelli $A$, et al. The health economics of bladder cancer. Pharmacoeconomics 2003;21:1315-1330.

2. Howlader $N$, Noone $A$, Krapcho $M$, et al. SEER cancer statistics review, 1975-2017. Bethesda, MD: National Cancer Institute 2019:1423-1437.

3. Nielsen $M E$, Smith $A B$, Meyer $A M$, et al. Trends in stage-specific incidence rates for urothelial carcinoma of the bladder in the United States: 1988 to 2006. Cancer 2014;120:86-95.

4. Chang SS, Boorjian SA, Chou R, et al. Diagnosis and treatment of non-muscle invasive bladder cancer: AUA/SUO guideline. J Urol 2016;196:1021-1029.

5. Martin-Doyle W, Leow JJ, Orsola A, et al. Improving selection criteria for early cystectomy in high-grade t1 bladder cancer: a meta-analysis of 15,215 patients. J Clin Oncol 2015;33:643-650.

6. Sylvester RJ, van der Meijden AP, Oosterlinck W, et al. Predicting recurrence and progression in individual patients with stage $\mathrm{Ta} T 1$ bladder cancer using EORTC risk tables: a combined analysis of 2596 patients from seven EORTC trials. Eur Urol 2006;49:466-477.

7. Babjuk $M$, Böhle $A$, Burger $M$, et al. EAU guidelines on non-muscleinvasive urothelial carcinoma of the bladder: update 2016. Eur Urol 2017;71:447-461.

8. Holmang $\mathrm{S}$, Hedelin $\mathrm{H}$, Anderstrom $\mathrm{C}$, et al. The importance of the depth of invasion in stage T1 bladder carcinoma: a prospective cohort study. J Urol 1997;157:800-804.

9. Millan-Rodriguez F, Chechile-Toniolo G, Salvador-Bayarri J, et al. Multivariate analysis of the prognostic factors of primary superficial bladder cancer. J Urol 2000;163:73-78.

10. van Rhijn BW, Liu L, Vis AN, et al. Prognostic value of molecular markers, sub-stage and European Organisation for the Research and Treatment of Cancer risk scores in primary T1 bladder cancer. BJUI 2012;110:1169-1176.

11. Choi W, Porten S, Kim S, et al. Identification of distinct basal and luminal subtypes of muscle-invasive bladder cancer with different sensitivities to frontline chemotherapy. Cancer Cell 2014;25:152-165.

12. McConkey DJ, Choi W, Ochoa A, et al. Therapeutic opportunities in the intrinsic subtypes of muscle-invasive bladder cancer. Hematol Oncol Clin 2015;29:377-394.

13. Cumberbatch MG, Foerster B, Catto JW, et al. Repeat transurethral resection in non-muscle-invasive bladder cancer: a systematic review. Eur Urol 2018;73:925-933.

14. Babjuk M, Burger $M$, Compérat EM, et al. European association of urology guidelines on non-muscle-invasive bladder cancer (TaT1 and carcinoma in situ)-2019 update. Eur Urol 2019;76:639-657.

15. Lamm DL, Blumenstein BA, Crissman JD, et al. Maintenance bacillus Calmette-Guerin immunotherapy for recurrent TA, T1 and carcinoma in situ transitional cell carcinoma of the bladder: a randomized Southwest Oncology Group Study. J Urol 2000;163:1124-1129. 
16. Sylvester RJ, van der Meijden AP, Lamm DL. Intravesical bacillus Calmette-Guerin reduces the risk of progression in patients with superficial bladder cancer: a meta-analysis of the published results of randomized clinical trials. J Urol 2002;168:1964-1970.

17. van Rhijn BW, Burger M, Lotan Y, et al. Recurrence and progression of disease in non-muscle-invasive bladder cancer: from epidemiology to treatment strategy. Eur Urol 2009;56:430-442.

18. Witjes JA, Hendricksen K. Intravesical pharmacotherapy for nonmuscle-invasive bladder cancer: a critical analysis of currently available drugs, treatment schedules, and long-term results. Eur Urol 2008;53:45-52.

19. Brake $M$, Loertzer $H$, Horsch $R$, et al. Long-term results of intravesical bacillus Calmette-Guerin therapy for stage T1 superficial bladder cancer. Urology 2000;55:673-678.

20. Novotny V, Froehner M, Ollig J, et al. Impact of adjuvant intravesical Bacillus Calmette-Guerin treatment on patients with high-grade T1 bladder cancer. Urol Int 2016;96:136-141.

21. Stimson C, Chang SS, Barocas DA, et al. Early and late perioperative outcomes following radical cystectomy: 90-day readmissions, morbidity and mortality in a contemporary series. J Urol 2010;184:1296-1300.

22. Joudi FN, Smith BJ, O'Donnell MA, et al. Contemporary management of superficial bladder cancer in the United States: a pattern of care analysis. Urology 2003;62:1083-1088.

23. Herr HW, Sogani PC. Does early cystectomy improve the survival of patients with high risk superficial bladder tumors? J Urol 2001;166:1296-1299.

24. Jäger W, Thomas C, Haag S, et al. Early vs delayed radical cystectomy for 'high-risk'carcinoma not invading bladder muscle: delay of cystectomy reduces cancer-specific survival. BJUI 2011;108:E284-E288.

25. Raj GV, Herr H, Serio AM, et al. Treatment paradigm shift may improve survival of patients with high risk superficial bladder cancer. J Urol 2007;177:1283-1286.

26. Denzinger S, Fritsche HM, Otto $W$, et al. Early versus deferred cystectomy for initial high-risk pT1G3 urothelial carcinoma of the bladder: do risk factors define feasibility of bladder-sparing approach? Eur Urol 2008;53:146-152.

27. Hautmann RE, Volkmer BG, Gust K. Quantification of the survival benefit of early versus deferred cystectomy in high-risk non-muscle invasive bladder cancer (T1 G3). World J Urol 2009;27:347-351.

28. Stöckle $M$, Alken $P$, Engelmann $U$, et al. Radical cystectomy-often too late? Eur Urol 1987;13:361-367.

29. Shen $P L$, lin ME, Hong $Y K$, et al. Bladder preservation approach versus radical cystectomy for high-grade non-muscle-invasive bladder cancer: a meta-analysis of cohort studies. World J Surg Oncol 2018;16:197.

30. Chang SS, Bochner BH, Chou R, et al. Treatment of non-metastatic muscle-invasive bladder cancer: AUA/ASCO/ASTRO/SUO guideline. J Urol 2017;198:552-559.

31. De Berardinis E, Busetto G, Antonini G, et al. T1G3 high-risk NMIBC (non-muscle invasive bladder cancer): conservative treatment versus immediate cystectomy. Int Urol Nephrol 2011;43:1047-1057.

32. Chang SS, Cookson MS. Non-muscle-invasive bladder cancer: the role of radical cystectomy. Urology 2005;66:917-922.

33. Freeman JA, Esrig D, Stein JP, et al. Radical cystectomy for high risk patients with superficial bladder cancer in the era of orthotopic urinary reconstruction. Cancer 1995;76:833-839.

34. Liaw CW, Winoker JS, Wiklund $P$, et al. The clinical and economic burden of perioperative complications of radical cystectomy. Transl Androl Urol 2019;8:S277-S279.

35. Stein JP, Lieskovsky G, Cote R, et al. Radical cystectomy in the treatment of invasive bladder cancer: long-term results in 1,054 patients. J Clin Oncol 2001;19:666-675.
36. Yang $L S$, Shan $B L$, Shan $L L$, et al. A systematic review and metaanalysis of quality of life outcomes after radical cystectomy for bladder cancer. Surg Oncol 2016;25:281-297.

37. Kulkarni GS, Finelli A, Fleshner NE, et al. Optimal management of high-risk T1G3 bladder cancer: a decision analysis. PLoS Med 2007; 4:e284.

38. Fernandez-Gomez J, Solsona E, Unda M, et al. Prognostic factors in patients with non-muscle-invasive bladder cancer treated with bacillus Calmette-Guérin: multivariate analysis of data from four randomized CUETO trials. Eur Urol 2008;53:992-1002.

39. Xylinas E, Kent M, Kluth $L$, et al. Accuracy of the EORTC risk tables and of the CUETO scoring model to predict outcomes in nonmuscle-invasive urothelial carcinoma of the bladder. $\mathrm{Br}$ J Cancer 2013; 109:1460-1466.

40. Parizi MK, Enikeev D, Glybochko PV, et al. Prognostic value of $\mathrm{T1}$ substaging on oncological outcomes in patients with non-muscleinvasive bladder urothelial carcinoma: a systematic literature review and meta-analysis. World J Urol 2020;38:1437-1449.

41. Rouprêt $M$, Seisen $T$, Compérat $E$, et al. Prognostic interest in discriminating muscularis mucosa invasion ( $T 1$ a vs $T 1 b$ ) in nonmuscle invasive bladder carcinoma: French national multicenter study with central pathology review. J Urol 2013;189:2069-2076.

42. Kitamura $H$, Kakehi $Y$. Treatment and management of high-grade T1 bladder cancer: what should we do after second TUR? Jpn J Clin Oncol 2015;45:315-322.

43. Amin MB, Greene FL, Edge SB, et al. The eighth edition AJCC cancer staging manual: continuing to build a bridge from a populationbased to a more "personalized" approach to cancer staging. CA Cancer J Clin 2017;67:93-99.

44. Bernardini S, Billerey $C$, Martin $M$, et al. The predictive value of muscularis mucosae invasion and p53 over expression on progression of stage T1 bladder carcinoma. J Urol 2001;165:42-46.

45. Ro JY, Ayala AG, El-Naggar A. Muscularis mucosa of urinary bladder Importance for staging and treatment. Am J Surg Pathol 1987; 11:668-669.

46. Leivo MZ, Sahoo D, Hamilton Z, et al. Analysis of T1 Bladder Cancer on Biopsy and Transurethral Resection Specimens: Comparison and Ranking of T1 Quantification Approaches to Predict Progression to Muscularis Propria Invasion. Am J Surg Pathol 2018;42:e1-e10.

47. Aziz A, Shariat SF, Roghmann F, et al. Prediction of cancer-specific survival after radical cystectomy in pT4a urothelial carcinoma of the bladder: development of a tool for clinical decision-making. BJUI 2016;117:272-279.

48. Berman DM, Kawashima A, Peng $Y$, et al. Reporting trends and prognostic significance of lymphovascular invasion in muscleinvasive urothelial carcinoma: A population-based study. Int J Urol 2015;22:163-170.

49. Mathieu R, Lucca I, Rouprêt $M$, et al. The prognostic role of lymphovascular invasion in urothelial carcinoma of the bladder. Nat Rev Urol 2016;13:471-479.

50. Kim HS, Kim M, Jeong CW, et al. Presence of lymphovascular invasion in urothelial bladder cancer specimens after transurethral resections correlates with risk of upstaging and survival: a systematic review and meta-analysis. Urol Oncol-Semin Ori Investig 2014;32:1191-1199.

51. Olsson H, Hultman P, Rosell J, et al. Population-based study on prognostic factors for recurrence and progression in primary stage T1 bladder tumours. Scand J Urol 2013;47:188-195.

52. Tilki D, Shariat SF, Lotan $Y$, et al. Lymphovascular invasion is independently associated with bladder cancer recurrence and survival in patients with final stage T1 disease and negative lymph nodes after radical cystectomy. BJUI 2013;111:1215-1221.

53. Shariat SF, Bolenz C, Godoy G, et al. Predictive value of combined immunohistochemical markers in patients with pT1 urothelial carcinoma at radical cystectomy. J Urol 2009;182:78-84.

54. Gakis G, Todenhöfer T, Braun M, et al. Immunohistochemical assessment of lymphatic and blood vessel invasion in T1 urothelial carcinoma of the bladder. Scand J Urol 2015;49:382-387. 
55. Resnick MJ, Bergey M, Magerfleisch L, et al. Longitudinal evaluation of the concordance and prognostic value of lymphovascular invasion in transurethral resection and radical cystectomy specimens. BJUI 2011;107:46-52.

56. Streeper NM, Simons CM, Konety BR, et al. The significance of lymphovascular invasion in transurethral resection of bladder tumour and cystectomy specimens on the survival of patients with urothelial bladder cancer. BJUI 2009; 103:475-479.

57. Mari A, Kimura S, Foerster B, et al. A systematic review and metaanalysis of the impact of lymphovascular invasion in bladder cancer transurethral resection specimens. BJUI 2019;123:11-21.

58. Masood S, Sriprasad S, Palmer J, et al. T1G3 bladder cancerindications for early cystectomy. Int Urol Nephrol 2003;36:41-44.

59. Yafi FA, Aprikian AG, Chin JL, et al. Impact of concomitant carcinoma in situ on upstaging and outcome following radical cystectomy for bladder cancer. World J Urol 2014;32:1295-1301.

60. Cookson MS, Herr HW, Zhang ZF, et al. The treated natural history of high risk superficial bladder cancer: 15-year outcome. J Urol 1997; 158:62-67.

61. Farrow GM, Utz DC, Rife CC, et al. Clinical observations on sixtynine cases of in situ carcinoma of the urinary bladder. Cancer Res 1977;37:2794-2798.

62. Lee DJ, Chang SS. The Role and Importance of Timely Radical Cystectomy for High-Risk Non-muscle-Invasive Bladder Cancer. Cancer Treat Res 2018;175:193-214.

63. Hassan JM, Cookson MS, Smith JA, et al. Outcomes in patients with pathological carcinoma in situ only disease at radical cystectomy. J Urol 2004;172:882-884.

64. Tilki D, Reich O, Svatek RS, et al. Characteristics and outcomes of patients with clinical carcinoma in situ only treated with radical cystectomy: an international study of 243 patients. J Urol 2010;183:1757-1763.

65. Amini E, Ahmadi N, Clifford TG, et al. Long term oncologic outcome in patients with bladder cancer after radical cystectomy: Impact of carcinoma in situ in the era of neoadjuvant chemotherapy. Int Urol Nephrol 2019;51:435-441.

66. Shapur NK, Katz R, Pode D, et al. Is radical cystectomy mandatory in every patient with variant histology of bladder cancer. Rare tumors 2011;3:67-70.

67. Matulay JT, Narayan VM, Kamat AM. Clinical and genomic considerations for variant histology in bladder cancer. Curr Oncol Rep 2019;21:23.

68. Shah RB, Montgomery JS, Montie JE, et al. Variant (divergent) histologic differentiation in urothelial carcinoma is under-recognized in community practice: impact of mandatory central pathology review at a large referral hospital. Urol Oncol-Semin Ori Investig 2013;31:1650-1655.

69. Kamat AM, Gee JR, Dinney CP, et al. The case for early cystectomy in the treatment of nonmuscle invasive micropapillary bladder carcinoma. J Urol 2006;175:881-885.

70. Weizer AZ, Wasco MJ, Wang R, et al. Multiple adverse histological features increase the odds of under staging T1 bladder cancer. J Urol 2009;182:59-65.

71. Ploeg M, Aben KK, Hulsbergen-van de Kaa CA, et al. Clinical epidemiology of nonurothelial bladder cancer: analysis of the Netherlands Cancer Registry. J Urol 2010;183:915-920.

72. Willis DL, Porten SP, Kamat AM. Should histologic variants alter definitive treatment of bladder cancer? Curr Opin Urol 2013;23:435-443.

73. Domanowska E, Jozwicki W, Domaniewski J, et al. Muscle-invasive urothelial cell carcinoma of the human bladder: multidirectional differentiation and ability to metastasize. Hum Pathol 2007;38:741-746.

74. Mally AD, Tin AL, Lee JK, et al. Clinical outcomes of patients with T1 nested variant of urothelial carcinoma compared to pure urothelial carcinoma of the bladder. Clin Genitourin Cancer 2018;16:e23-e27.
75. Willis D, Kamat AM. Nonurothelial bladder cancer and rare variant histologies. Hematol Oncol Clin 2015;29:237-252.

76. Sui W, Matulay JT, James MB, et al. Micropapillary bladder cancer: insights from the National Cancer Database. Bladder Cancer 2016;2:415-423.

77. Vetterlein MW, Wankowicz SA, Seisen T, et al. Neoadjuvant chemotherapy prior to radical cystectomy for muscle-invasive bladder cancer with variant histology. Cancer 2017;123:4346-4355.

78. Shariat SF, Tokunaga H, Zhou J, et al. p53, p21, pRB, and p16 expression predict clinical outcome in cystectomy with bladder cancer. J Clin Oncol 2004;22:1014-1024.

79. van Rhijn BW, Catto JW, Goebell PJ, et al. Molecular markers for urothelial bladder cancer prognosis: toward implementation in clinical practice. Urol Oncol-Semin Ori Investig 2014;32:1078-1087.

80. Dalbagni G, Parekh DJ, Ben-Porat L, et al. Prospective evaluation of p53 as a prognostic marker in T1 transitional cell carcinoma of the bladder. BJUI 2007;99:281-285.

81. Desgrandchamps F, LeMaoult J, Goujon A, et al. Prediction of non-muscle-invasive bladder cancer recurrence by measurement of checkpoint HLAG's receptor ILT2 on peripheral CD8+ T cells. Oncotarget 2018;9:33160-33169.

82. Knowles MA. Molecular subtypes of bladder cancer: Jekyll and Hyde or chalk and cheese? Carcinogenesis 2006;27:361-373.

83. Lelo A, Prip F, Harris BT, et al. STAG2 Is a Biomarker for Prediction of Recurrence and Progression in Papillary Non-Muscle-Invasive Bladder Cancer. Clin Cancer Res 2018;24:4145-4153.

84. Passoni N, Gayed B, Kapur P, et al. Cell-cycle markers do not improve discrimination of EORTC and CUETO risk models in predicting recurrence and progression of non-muscle-invasive high-grade bladder cancer. Urol Oncol-Semin Ori Investig 2016;34:485.e7-485.e14.

85. Breyer J, Otto W, Wirtz RM, et al. ERBB2 expression as potential riskstratification for early cystectomy in patients with pT1 bladder cancer and concomitant carcinoma in situ. Urol Int 2017;98:282-289.

86. Fristrup N, Birkenkamp-Demtröder K, Reinert T, et al. Multicenter validation of Cyclin D1, MCM7, TRIM29, and UBE2C as prognostic protein markers in non-muscle-invasive bladder cancer. Am J Pathol 2013;182:339-349.

87. van Kessel KE, van der Keur KA, Dyrskjøt L, et al. Molecular markers increase precision of the european association of urology nonmuscle-invasive bladder cancer progression risk groups. Clin Cancer Res 2018;24:1586-1593.

88. Goebell PJ, Groshen SG, Schmitz-Dräger BJ, et al. p53 immunohistochemistry in bladder cancer-a new approach to an old question. Urol Oncol-Semin Ori Investig 2010;28:377-388.

89. Shariat SF, Weizer AZ, Green A, et al. Prognostic value of P53 nuclear accumulation and histopathologic features in $\mathrm{T} 1$ transitional cell carcinoma of the urinary bladder. Urology 2000;56:735-740.

90. van Rhijn BW, Vis AN, van der Kwast TH, et al. Molecular grading of urothelial cell carcinoma with fibroblast growth factor receptor 3 and MIB-1 is superior to pathologic grade for the prediction of clinical outcome. J Clin Oncol 2003;21:1912-1921.

91. Chandrasekar T, Zlotta A, Shen J, et al. RNA sequencing identifies 3 different molecular grades and immune checkpoint cascades with distinct clinical behaviour in non-muscle invasive bladder cancer. Eur Urol Suppl 2018;17:e1332.

92. Burger M, van der Aa MN, van Oers JM, et al. Prediction of progression of non-muscle-invasive bladder cancer by WHO 1973 and 2004 grading and by FGFR3 mutation status: a prospective study. Eur Urol 2008;54:835-844.

93. van Rhijn BW, Zuiverloon TC, Vis AN, et al. Molecular grade (FGFR3/ MIB-1) and EORTC risk scores are predictive in primary non-muscleinvasive bladder cancer. Eur Urol 2010;58:433-441.

94. Shariat SF, Ashfaq R, Sagalowsky Al, et al. Predictive value of cell cycle biomarkers in nonmuscle invasive bladder transitional cell carcinoma. J Urol 2007; 177:481-487. 
95. Damrauer JS, Hoadley KA, Chism DD, et al. Intrinsic subtypes of highgrade bladder cancer reflect the hallmarks of breast cancer biology. Proc Natl Acad Sci 2014;111:3110-3115.

96. Rebola J, Aguiar P, Blanca A, et al. Predicting outcomes in non-muscle invasive (Ta/T1) bladder cancer: the role of molecular grade based on luminal/basal phenotype. Virchows Arch 2019;475:445-455.

97. Groenendijk FH, de Jong J, van de Putte EEF, et al. ERBB2 mutations characterize a subgroup of muscle-invasive bladder cancers with excellent response to neoadjuvant chemotherapy. Eur Urol 2016;69:384-388.

98. Network CGAR. Comprehensive molecular characterization of urothelial bladder carcinoma. Nature 2014;507:315-322.
99. Powles T, Eder JP, Fine GD, et al. MPDL3280A (anti-PD-L1) treatment leads to clinical activity in metastatic bladder cancer. Nature 2014; 515:558-562.

100. Rebouissou S, Bernard-Pierrot I, de Reyniès A, et al. EGFR as a potential therapeutic target for a subset of muscle-invasive bladder cancers presenting a basal-like phenotype. Sci Transl Med 2014;6:244ra91.

101. Werntz RP, Adamic B, Steinberg GD. Emerging therapies in the management of high-risk non-muscle invasive bladder cancer (HRNMIBC). World J Urol 2019;37:2031-2040. 